\title{
Microenvironmental InterFereNce of metabolism regulates chemosensitivity
}

Cell Research (2016) 26:867-868. doi:10.1038/cr.2016.82; published online 28 June 2016

The tumor microenvironment is recognized as a critical regulator of cancer progression, and multiple roles are also emerging for the microenvironment in modulating response to therapeutic intervention. A recent study by Wang and colleagues identified IFN- $\gamma$ as a central effector of $\mathrm{CD8}^{+}$ $T$ cell-mediated regulation of glutathione and cysteine metabolism in fibroblasts, which consequently abrogates stromal-induced resistance through modulation of cisplatin intracellular content in ovarian cancer cells.

While most chemotherapeutic agents are initially effective at debulking tumors, the emergence of resistance is commonly observed. This can arise from the accumulation of genetic and epigenetic alterations in malignant cells, but also through dynamic and reciprocal interactions between cancer cells and their surrounding environment.

The tumor microenvironment (TME) comprises multiple non-cancerous cell types, including infiltrating leukocytes, endothelial cells, and stromal fibroblasts. Depending on the stage of tumor progression and the particular organ site, TME cells can have tumor-suppressive or tumor-promoting roles, driven in part via their 'education' by evolving cancer cells. Interestingly, many of the same mechanisms through which the TME promotes malignant progression are also "co-opted" in the context of therapy resistance [1].

In advanced ovarian cancers, the stroma comprises up to $50 \%$ of the tumor mass [2], with cancer-associated fibroblasts (CAFs) representing a major cellular constituent. CAFs have been shown to decrease sensitivity of diverse cancer cell types to various anticancer drugs, through the production of secretory molecules such as $\operatorname{HGF}[3,4]$. CAFs can also alter the physical properties of the tumor through the excessive deposition and aberrant remodeling of the extracellular matrix, which can contribute to formation of an interstitial barrier that prevents efficient drug delivery. Consequently, targeting CAFs leads to increased bioavailability of chemotherapeutics including doxorubicin [5], and enables immunological detection and destruction of the tumor [6]. However, depleting specific CAF populations may outweigh these beneficial effects in certain contexts, favoring the development of more aggressive and less differentiated tumors.

The study by Wang and colleagues [7] further expands the range of mechanisms used by CAFs to mediate chemotherapeutic resistance by identifying a novel role for CAFs in decreasing cisplatin levels within treated ovarian cancer cells (Figure 1). The intriguing finding that this occurs via alterations of specific metabolites within the ovarian TME raises the possibility of targeting metabolic pathways in combination with chemotherapy to enhance therapeutic response. It has previously been reported that a switch to aerobic glycolysis occurs in CAFs (which is termed the "reverse Warburg effect") to generate lactate and ketones. Once internalized, these molecules can act as onco-metabolites, fueling oxidative mitochondrial metabolism in cancer cells [8]. Wang et al. [7] now show that through glutathione (GSH) and cysteine secretion, CAFs limit the levels of intracellular platinum in cancer cells, thereby reducing DNA damage and cisplatin-induced apoptosis. The presence of cystine (the oxidized dimeric form of cysteine) in the extracellular milieu is necessary for high levels of GSH and cysteine to be released specifically from CAFs. However, whether the increased metabolic processing of cystine into thiols is specific to CAFs compared to other stromal or immune cell types remains to be determined, as does the source of high cystine content within the ovarian TME.

The authors also explored how CAFs may interact with additional cell types in the ovarian TME, and focused on $\mathrm{CD}^{+}$ $\mathrm{T}$ cells. As for many solid tumors, the presence of intra-tumoral $\mathrm{T}$ cells correlates with improved clinical outcome in advanced ovarian carcinoma [9]. Previous studies in pancreatic cancer have shown that targeting fibroblast-derived CXCL12 increased T cell accumulation and sensitized pancreatic tumors to immune checkpoint blockade [10]. Wang et al. hypothesized that $\mathrm{CD}^{+} \mathrm{T}$ cells could also be harnessed in order to block fibroblast-mediated chemoresistance. This was confirmed by the identification of the IFN- $\gamma /$ IFN- $\gamma$ R/STAT1 signaling pathway as a regulator of thiol and GSH metabolism in CAFs (Figure 1). The specificity of action involves a direct transcriptional repression of the $x C T$ gene, mediated by STAT1, a downstream effector of IFN- $\gamma$ signaling. $x C T$ encodes the light chain component of the xctransporter that controls cystine import into the cell. Transcriptional repression of $x C T$, which alters the production and release of thiols, and consequently the level of cisplatin-DNA adducts in tumor 


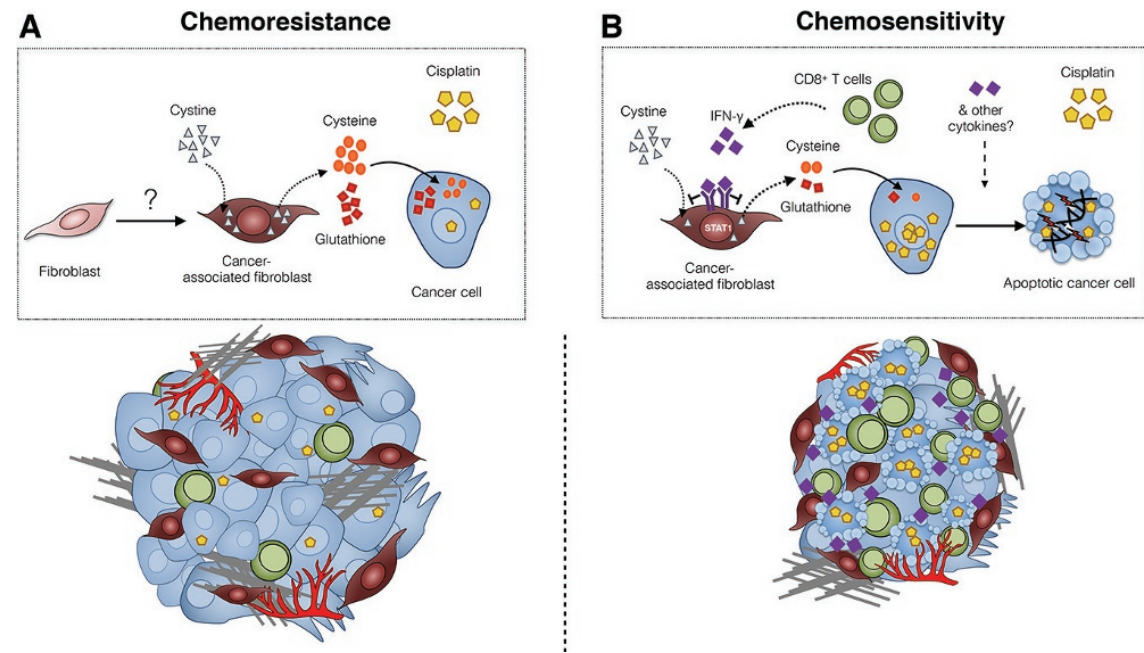

Figure 1 Mechanisms of chemoresistance in the ovarian cancer microenvironment, and strategies to overcome resistance. (A) Within ovarian TME, fibroblasts are converted into CAFs by a yet unknown mechanism. CAFs utilize cystine to generate cysteine and glutathione, which are then released into the extracellular milieu. The uptake of glutathione and cysteine by tumor cells leads to elevated intracellular glutathione level. As a consequence, levels of intracellular cisplatin are decreased, resulting in lower levels of DNA damage and cell death, and cisplatin resistance. This chemoresistant stroma comprises a high number of CAFs and a limited number of infiltrated $\mathrm{CD} 8^{+} \mathrm{T}$ lymphocytes. (B) $\mathrm{CD} 8^{+} \mathrm{T}$ cell-derived IFN- $\gamma$ activates the IFN- $\gamma \mathrm{R} 1 / \mathrm{STAT} 1$ signaling pathway in CAFs, leading to reduction of extracellular cystine consumption, downregulation of cysteine synthesis and degradation of glutathione. Through this mechanism, intracellular cisplatin accumulation is restored in ovarian cancer cells, leading to ovarian cancer cell apoptosis. This chemosensitive stroma comprises high number of CD8+ lymphocytes, which predict responsiveness to cisplatin independently of CAF content.

cells, was only observed in CAFs and not in cancer cells. Whether other normal cell types in the TME are similarly capable of regulating thiol metabolism when exposed to IFN- $\gamma$ was not determined, and would now be interesting to investigate.

Importantly, the authors report that high stromal content was found in chemoresistant ovarian cancer patients and correlated with shorter overall survival. However, by comparing survival of patients in which tumors presented an increased number of smooth muscle actin $(\mathrm{SMA})^{+}$fibroblasts and either high or low number of $\mathrm{CD} 8^{+} \mathrm{T}$ cells, the authors suggest that independent of stromal content, cisplatin-based therapy would be efficacious as long as $\mathrm{CD} 8^{+} \mathrm{T}$ cells infiltrate into the tumor mass. Tumor control by $\mathrm{T}$ cells is often mediated via classical perforin- and granzyme-mediated lysis. Additionally, it is now well established that Th1 cells secrete both IFN- $\gamma$ and TNF- $\alpha$ which can have pleiotropic effects including mediating the activation of
CTLs, acting on other cells in the TME, promoting the emergence of anti-tumoral macrophages, and directly affecting cancer cells themselves. Thus, while the mechanisms of tumor resistance reported by Wang et al., which focus on metabolic alteration of CAF-mediated chemoresistance, differ from conventional paracrine regulation of the immune response, a common feature is the central role of effector $\mathrm{CD}^{+} \mathrm{T}$ cells in TME. It will be important to determine whether the regulation of tumor cell DNA damage by $\mathrm{CD} 8^{+} \mathrm{T}$ cell-mediated control of $\mathrm{CAF}$ metabolism could affect other agents causing DNA interstrand crosslinking in addition to cisplatin.

Immunotherapy is becoming a mainstay of cancer therapy in several tumor types including ovarian cancer, and the novel mechanisms of resistance to cisplatin chemotherapy identified by Wang and colleagues provide additional avenues for therapeutic targeting of pro-tumorigenic $\mathrm{CAF}$ functions through $\mathrm{CD} 8^{+} \mathrm{T}$ cell modulation. A number of interesting questions remain, however, which will be important to address to determine whether this resistance mechanism can be fully circumvented. First, what is the trigger that drives high thiol metabolism in CAFs, and is it dependent on a permissive TME? Second, what regulates the presence of $\mathrm{CD} 8^{+} \mathrm{T}$ cells in the ovarian TME, and can this be manipulated to increase T cell infiltration? Third, is the DNA repair signaling pathway in ovarian cancer cells independently affected by IFN- $\gamma$ ? Fourth, how stable are the effects of $\mathrm{T}$ cell-derived IFN- $\gamma$ on fibroblast metabolism and consequently, on tumor cell DNA damage? As we gain insights into how interactions within the TME contribute to drug resistance and tumor recurrence, further studies examining the interaction between the CAF niche and adaptive immune cells could offer additional strategies to adoptive $\mathrm{T}$ cell therapy or fibroblast depletion as a mean to enhance the efficacy of DNA damaging agents.

\section{Leila Akkari ${ }^{1,2}$, Johanna A Joyce ${ }^{1,2}$}

${ }^{1}$ Ludwig Institute for Cancer Research, Lausanne, Switzerland; ${ }^{2}$ Department of Oncology, University of Lausanne, Lausanne, Switzerland Correspondence: Johanna A Joyce E-mail: johanna@joycelab.org

\section{References}

1 Klemm F, Joyce JA. Trends Cell Biol 2015; 25:198-213.

2 Labiche A, Heutte N, Herlin P, et al. Int J Gynecol Cancer 2010; 20:28-33.

3 Straussman R, Morikawa T, Shee K, et al. Nature 2012; 487:500-504.

4 Wilson TR, Fridlyand J, Yan Y, et al. Nature 2012; 487:505-509.

5 Olive KP, Jacobetz MA, Davidson CJ, et al. Science 2009; 324:1457-1461.

6 Kraman M, Bambrough PJ, Arnold JN, et al. Science 2010; 330:827-830.

7 Wang W, Kryczek I, Dostál L, et al. Cell 2016; 165:1092-1105.

8 Sotgia F, Whitaker-Menezes D, MartinezOutschoorn UE, et al. Cell Cycle 2012; 11:4390-4401.

9 Zhang L, Conejo-Garcia JR, Katsaros D, et al. N Engl J Med 2003; 348: 203-213.

10 Feig C, Jones JO, Kraman M, et al. Proc Natl Acad Sci USA 2013; 110:20212-20217. 